\title{
Convertible Bond Arbitrage Study
}

\author{
Tim Xiao
}

\begin{abstract}
In a convertible bond arbitrage strategy, the arbitrageur purchases a convertible bond and sells the underlying stock to create a delta neutral position. The number of shares sold short usually reflects a deltaneutral or market neutral ratio. People believe that convertible arbitrage is mainly due to convertible underpricing. Empirically, however, we do not find evidence supporting the underpricing hypothesis. Instead, we find that convertibles have relatively large positive gammas. As a typical convertible arbitrage strategy employs delta-neutral hedging, a large positive gamma can make the portfolio highly profitable, especially for a large movement in the underlying stock price.
\end{abstract}

Key Words: hybrid financial instrument, convertible bond, convertible underpricing, convertible arbitrage, default time approach, default probability approach, jump diffusion. 


\section{Introduction}

A company can raise capital in financial markets either by issuing equities, bonds, or hybrids (such as convertible bonds). From an investor's perspective, convertible bonds with embedded optionality offer certain benefits of both equities and bonds - like the former, they have the potential for capital appreciation and like the latter, they offer interest income and safety of principal. The convertible bond market is of primary global importance.

Tsiveriotis and Fernandes (1998) argue that in practice one is usually uncertain as to whether the bond will be converted, and thus propose dividing convertible bonds into two components: a bond part that is subject to credit risk and an equity part that is free of credit risk. A simple description of this model and an easy numerical example in the context of a binomial tree can be found in Hull (2003).

The jump-diffusion model was first introduced by Merton (1976) in the market risk context for modeling asset price behavior that incorporates small day-to-day diffusive movements together with larger randomly occurring jumps. Over the last decade, people attempt to propagate the model from the market risk domain to the credit risk arena. At the heart of the jump-diffusion models lies the assumption that the total expected rate of return to the stockholders is equal to the risk-free interest rate under a risk-neutral measure.

Because of their hybrid nature, convertible bonds attract different type of investors. Especially, convertible arbitrage hedge funds play a dominant role in primary issues of convertible debt. In fact, it is believed that hedge funds purchase $70 \%$ to $80 \%$ of the convertible debt offered in primary markets. A prevailing belief in the market is that convertible arbitrage is mainly due to convertible underpricing (i.e., the model prices are on average higher than the observed trading prices) (see Ammann et al (2003), Calamos (2011), Choi et al (2009), Loncarski et al (2009), etc.). However, Agarwal et al (2007) and Batta et al (2007) argue that the excess returns from convertible arbitrage strategies are not mainly due to underpricing, but rather partly due to illiquid. Calamos (2011) believes that arbitrageurs in general take advantage of volatility. A higher volatility in the underlying equity translates into a higher value of the equity option and a lower 
conversion premium. Multiple views reveal the complexity of convertible arbitrage, involving taking positions in the convertible bond and the underlying asset that hedges certain risks but leaves managers exposed to other risks for which they reap a reward.

We model both equities and bonds as defaultable in a consistent way. When a firm goes bankrupt, the investors who take the least risk are paid first. Secured creditors have the best chances of seeing the value of their initial investments come back to them. Bondholders have a greater potential for recovering some their losses than stockholders who are last in line to be repaid and usually receive little, if anything. The default proceedings provide a justification for our modeling assumptions: Different classes of securities issued by the same company have the same default probability but different recovery rates. Given this model, we are able to back out the market prices.

Using the model proposed, we conduct an empirical study of convertible bonds. We obtain a data set from FinPricing (2013). The data set contains 164 convertible bonds and 2 years of daily market prices as well as associated interest rate curves, credit curves, stock prices, implied Black-Scholes volatilities and recovery rates.

The empirical results show that the model prices fluctuate randomly around the market prices, indicating the model is quite accurate. Our empirical evidence does not support a systematic underpricing hypothesis. A similar conclusion is reached by Ammann et al (2008) who use a Monte-Carlo simulation approach. Moreover, market participants almost always calibrate their models to the observed market prices using implied convertible volatilities. Therefore, underpricing may not be the main driver of profitability in convertible arbitrage.

We study the sensitivities of convertible bonds and find that convertible bonds have relatively large positive gammas, implying that convertible arbitrage can make a profit on a large upside or downside movement in the underlying stock price. Since convertible bonds are issued mainly by start-up or small companies (while more established firms rely on other means of financing), the chance of a large movement in either direction is very likely. Even for very small movements in the underlying stock price, profits can still be generated from the yield of the convertible bond and the interest rebate for the short position. 


\section{$2 \quad$ Model}

Convertible bonds can be thought of as normal corporate bonds with embedded options, which enable the holder to exchange the bond asset for the issuer's stock. Despite their popularity and ubiquity, convertible bonds still pose difficult modeling challenges, given their hybrid nature of containing both debt and equity features. Further complications arise due to the frequent presence of complex contractual clauses, such as, put, hard call, soft call, and other path-dependent trigger provisions. Contracts of such complexity can only be solved by numerical methods, such as, Monte Carlo simulation, tree/lattice approaches, or PDE solutions.

Three sources of randomness exist in a convertible bond: the stock price, the interest rate, and the credit spread. As practitioners tend to eschew models with more than two factors, it is a legitimate question: How can we reduce the number of factors or which factors are most important? Grimwood and Hodges (2002) conduct a sensitivity study and find that accurately modeling the equity process appears crucial. This is why all convertible bond models in the market capture, at a minimum, the dynamics of the underlying equity price. Since convertible bonds are issued mainly by start-up or small companies (while more established firms rely on other means of financing), credit risk plays an important role in the valuation. Grimwood and Hodges (2002) further note that the interest rate process is of second order importance. Similarly, Brennan and Schwartz (1980) conclude that the effect of a stochastic interest rate on convertible bond prices is so small that it can be neglected. Furthermore, Ammann et al (2008) notice that the overall pricing benefit of incorporating stochastic interest rates would be very limited and would not justify the additional computational costs. For these reasons, most practical convertible models in the market do not take stochastic interest rate into account.

The risk-free stock price process can be described as

$$
d S(t)=r(t) S(t) d t+\sigma S(t) d W(t)
$$


where $S(t)$ denotes the stock price, $r(t)$ denotes the risk-free interest rate, $\sigma$ denotes the volatility, $W(t)$ denotes a Wiener process.

The expectation of equation (1) is

$$
E\left(d S(t) \mid \mathcal{F}_{t}\right)=r(t) S(t) d t
$$

where $E\left\{\cdot \mid \mathcal{F}_{t}\right\}$ is the expectation conditional on the $\mathcal{F}_{t}$.

Next, we turn to a defaultable stock. The defaultable stock process proposed by Davis and Lischka (1999), Andersen and Buffum (2004), and Bloomberg (2009), etc., is given by

$$
d S(t)=(r(t)+h(t)) S\left(t_{-}\right) d t+\hat{O} S\left(t_{-}\right) d W(t)-S\left(t_{-}\right) d U(t)
$$

where $U(t)$ is an independent Poisson process with $d U(t)=1$ with probability $h(t) d t$ and 0 otherwise, $h(t)$ is the hazard rate or the default intensity, $S\left(t_{-}\right)$is the stock price immediately before any jump at time $t$. The expectation of $d U(t)$ is $E\left(d U(t) \mid \mathcal{F}_{t}\right)=h(t) d t$.

The expectation of equation (3) is given by

$$
E\left(d S(t) \mid \mathcal{F}_{t}\right)=(r(t)+h(t)) S(t) d t-S(t) h(t) d t=r(t) S(t) d t
$$

It is shown in equation (4) that the expected return of a defaultable stock under a jump-diffusion model also grows at the risk-free interest rate. Equation (3) is a simpler version of the Merton's Jumpdiffusion model where the number of jumps is 1 .

However, we wonder whether it is appropriate to propagate the jump-diffusion model directly from the market risk domain to the credit risk domain, as credit risk actually impacts the valuation of assets. This is why financial institutions are required by regulators to report CVA. In fact, we will show in the following derivation that the expected return of a defaultable asset under a risk-neutral measure is actually equal to a risky rate instead of the risk-free rate. This conclusion is very important for risky valuation.

In the reduced-form models, the stopping (or default) time $\tau$ of a firm is modeled as a Cox arrival process (also known as a doubly stochastic Poisson process) whose first jump occurs at default and is defined as, 


$$
\tau=\inf \left\{t: \int_{0}^{t} h\left(s, \Phi_{s}\right) d s \geq \Delta\right\}
$$

where $h(t)$ or $h\left(t, \Phi_{t}\right)$ denotes the stochastic hazard rate or arrival intensity dependent on an exogenous common state $\Phi_{t}$, and $\Delta$ is a unit exponential random variable independent of $\Phi_{t}$.

It is well-known that the survival probability from time $t$ to $s$ in this framework is defined by

$$
p(t, s):=P(\tau>s \mid \tau>t, Z)=\exp \left(-\int_{t}^{s} h(u) d u\right)
$$

The default probability for the period $(t, s)$ in this framework is given by

$$
q(t, s):=P(\tau \leq s \mid \tau>t, Z)=1-p(t, s)=1-\exp \left(-\int_{t}^{s} h(u) d u\right)
$$

We consider a defaultable asset that pays nothing between dates $t$ and $T$. Let $V(t)$ and $V(T)$ denote its values at $t$ and $T$, respectively. Risky valuation can be generally classified into two categories: the default time approach (DTA) and the default probability (intensity) approach (DPA).

Under a risk-neutral measure, the value of this defaultable asset is the discounted expectation of all the payoffs and is given by

$$
V(t)=E\left[\left(D(t, T) V(T) 1_{\tau>T}+D(t, \tau) \varphi V(\tau) 1_{\tau \leq T}\right) \mid \mathcal{F}_{t}\right]
$$

where $1_{Y}$ is an indicator function that is equal to one if $Y$ is true and zero otherwise, and $D(t, \tau)$ denotes the stochastic risk-free discount factor at $t$ for the maturity $\tau$ given by

$$
D(t, \tau)=\exp \left[-\int_{t}^{\tau} r(u) d u\right]
$$

Although the DTA is very intuitive, it has the disadvantage that it explicitly involves the default time/jump. We are very unlikely to have complete information about a firm's default point, which is often inaccessible. Moreover, in a derivative transaction, the market-value-at-default $V(\tau)$ usually reflects the 
replacement cost of the transaction, where the replacement is also defaultable ${ }^{1}$. Therefore $V(\tau)$ should be determined via another risky valuation and so forth. Usually, valuation under the DTA is performed via Monte Carlo simulation.

In our derivation, we use the approximation $\exp (y) \approx 1+y$ for very small $y$. The survival and default probabilities for the period $(t, t+\Delta t)$ are given by

$$
\begin{aligned}
& \hat{p}(t):=p(t, t+\Delta t)=\exp (-h(t) \Delta t) \approx 1-h(t) \Delta t \\
& \hat{q}(t):=q(t, t+\Delta t)=1-\exp (-h(t) \Delta t) \approx h(t) \Delta t
\end{aligned}
$$

Under a risk-neutral measure, the value of the asset at $t$ is the expectation of all the payoffs discounted at the risk-free rate and is given by

$$
V(t)=E\left\{\exp (-r(t) \Delta t)[\hat{p}(t)+\varphi(t) \hat{q}(t)] V(t+\Delta t) \mid \mathcal{F}_{t}\right\} \approx E\left\{\exp (-y(t) \Delta t) V(t+\Delta t) \mid \mathcal{F}_{t}\right\}
$$

where $y(t)=r(t)+h(t)(1-\varphi(t))=r(t)+c(t)$ denotes the risky rate and $c(t)=h(t)(1-\varphi(t))$ is called the (short) credit spread.

Similarly, we have

$$
V(t+\Delta t)=E\left\{\exp (-y(t+\Delta t) \Delta t) V(t+2 \Delta t) \mid \mathcal{F}_{t+\Delta t}\right\}
$$

Note that $\exp (-y(t) \Delta t)$ is $\mathcal{F}_{t+\Delta t}$-measurable. By definition, an $\mathcal{F}_{t+\Delta t}$-measurable random variable is a random variable whose value is known at time $t+\Delta t$. Based on the taking out what is known and tower properties of conditional expectation, we have

$$
\begin{aligned}
V(t) & =E\left\{\exp (-y(t) \Delta t) V(t+\Delta t) \mid \mathcal{F}_{t}\right\} \\
& =E\left\{\exp (-y(t) \Delta t) E\left[\exp (-y(t+\Delta t) \Delta t) V(t+2 \Delta t) \mid \mathcal{F}_{t+\Delta t}\right] \mid \mathcal{F}_{t}\right\} \\
& \left.=E\left\{\exp \left(-\sum_{i=0}^{1} y(t+i \Delta t) \Delta t\right)\right) V(t+2 \Delta t) \mid \mathcal{F}_{t}\right\}
\end{aligned}
$$

\footnotetext{
${ }^{1}$ Many people in the market use the risk-free value as the market-value-at-default, which is inappropriate as any contract in the OTC market is risky when taking counterparty risk into account.
} 
By recursively deriving from $t$ forward over $T$ and taking the limit as $\Delta t$ approaches zero, the risky value of the asset can be expressed as

$$
V(t)=E\left\{\exp \left[-\int_{t}^{T} y(u) d u\right] V(T) \mid \mathcal{F}_{t}\right\}
$$

Using the DPA, we obtain a closed-form solution for pricing an asset subject to credit risk. Another good example of the DPA is the CDS model proposed by J.P. Morgan (1999).

Under a risk-neutral measure the market price of risk and risk preferences are irrelevant to asset pricing (Hull, 2003) and thereby the expectation of a risk-free asset grows at the risk-free interest rate. However, credit risk actually has a significant impact on asset prices. This is the reason that regulators, such as IASB and BCBS, require financial institutions to report a CVA in addition to the risk-free MTM value to reflect credit risk.

If a company files bankruptcy, both bonds and stocks go into a default status. In other words, the default probabilities for both of them are the same (i.e., equal to the firm's probability of default). But the recovery rates are different because the stockholders are the lowest priority in the list of the stakeholders in the company, whereas the bondholders have a higher priority to receive a higher percentage of invested funds. The default proceedings provide a justification for our modeling assumptions: Different classes of securities issued by the same company have the same default probability but different recovery rates.

The stochastic differential equation (SDE) of a defaultable stock is defined as

$$
d S(t)=\left(r(t)+h(t)\left(1-\varphi_{s}(t)\right)\right) S(t) d t+\sigma S(t) d W(t)=y(t) S(t) d t+\sigma S(t) d W(t)
$$

where $\varphi_{s}$ is the recovery rate of the stock and $y(t)=r(t)+h(t)\left(1-\varphi_{s}(t)\right)$ is the risky rate.

For most practical problems, zero recovery at default (or jump to zero) is unrealistic. For example, the stock of Lehman Brothers fell 94.3\% on September 15, 2008 after the company filed for Chapter 11 bankruptcy. Similarly, the shares of General Motors (GM) plunged 32\% on June 1, 2009 after the firm initiated Chapter 11 bankruptcy. A good framework should flexibly allow people to incorporate different recovery assumptions into risky valuation. 
Equation (16) is the direct derivation of equation (15). The formula allows different assumptions concerning recovery on default. In particular, $\varphi_{s}=0$ represents the situation where the stock price jumps to 0 , and $\varphi_{s}=1$ corresponds to the risk-free case. The expectation of equations (16) is

$$
E\left(d S(t) \mid \mathcal{F}_{t}\right)=\left(r(t)+h(t)\left(1-\varphi_{s}(t)\right)\right) S(t) d t
$$

Equation (17) says that the expected return of a stock subject to credit risk is equal to a risky rate rather than the risk-free rate. The risky rate reflects the compensation investors receive for bearing credit risk.

\section{PDE Algorithm}

The numerical solution of our risky model can be obtained by either PDE methods, tree approaches, or Monte Carlo simulation. In this paper, we introduce the PDE procedure, but of course the methodology can be easily extended to the tree/lattice or Monte Carlo algorithms.

The defaultable stock price process is given by

$$
d S(t)=\left(r(t)-q(t)+h(t)\left(1-\varphi_{s}(t)\right)\right) S(t) d t+\sigma S(t) d W(t)=\mu(t) S(t) d t+\sigma S(t) d W(t)
$$

where $q(t)$ is the dividend and $\mu(t)=r(t)-q(t)+h(t)\left(1-\varphi_{s}(t)\right)$.

Suppose that $G(S, t)$ is some function of $S$ and $t$. Applying Ito Lemma, we have

$$
d G=\left(\mu S \frac{\partial G}{\partial S}+\frac{\partial G}{\partial t}+\frac{1}{2} \sigma^{2} S^{2} \frac{\partial^{2} G}{\partial S^{2}}\right) d t+\sigma S \frac{\partial G}{\partial S} d W
$$

Since the Wiener process underlying $S$ and $G$ are the same, we can construct the following portfolio so that the Wiener process can be eliminated.

$$
X=G-S \frac{\partial G}{\partial S}
$$

Therefore, we have

$$
d X=d G-\frac{\partial G}{\partial S} d S=\left(\frac{\partial G}{\partial t}+\frac{1}{2} \sigma^{2} S^{2} \frac{\partial^{2} G}{\partial S^{2}}\right) d t
$$


In contrast to all previous studies, we believe that the defaultable equity should grow at the risky rate of the equity including dividends, whereas the equity part of the convertible bond should earn the risky rate of the equity excluding dividends, i.e.,

$$
\left(r+h\left(1-\varphi_{s}\right)\right) G d t-\left(r-q+h\left(1-\varphi_{s}\right)\right) \frac{\partial G}{\partial S} S d t=d X=\left(\frac{\partial G}{\partial t}+\frac{1}{2} \sigma^{2} S^{2} \frac{\partial^{2} G}{\partial S^{2}}\right) d t
$$

So that the PDE of the equity component is given by

$$
\frac{\partial G}{\partial t}+\frac{1}{2} \sigma^{2} S^{2} \frac{\partial^{2} G}{\partial S^{2}}+\left(r-q+h\left(1-\varphi_{s}\right)\right) S \frac{\partial G}{\partial S}-\left(r+h\left(1-\varphi_{s}\right)\right) G=0
$$

Similarly applying Ito Lemma to the bond part of the convertible $B(S, t)$, we obtain

$$
d B=\left(\mu S \frac{\partial B}{\partial S}+\frac{\partial B}{\partial t}+\frac{1}{2} \sigma^{2} S^{2} \frac{\partial^{2} B}{\partial S^{2}}\right) d t+\sigma S \frac{\partial B}{\partial S} d W
$$

Let us construct a portfolio so that we can eliminate the Wiener process as follows

$$
Y=B-S \frac{\partial B}{\partial S}
$$

Thus, we have

$$
d Y=d B-\frac{\partial B}{\partial S} d S=\left(\frac{\partial B}{\partial t}+\frac{1}{2} \sigma^{2} S^{2} \frac{\partial^{2} B}{\partial S^{2}}\right) d t
$$

The defaultable equity should grow at the risky rate of the equity including dividends, while the bond part of the convertible bond grows at the risky rate of the bond. Consequently, we have

$$
\left(r+h\left(1-\varphi_{b}\right)\right) B d t-\left(r-q+h\left(1-\varphi_{s}\right)\right) \frac{\partial B}{\partial S} S d t=d Y=\left(\frac{\partial B}{\partial t}+\frac{1}{2} \sigma^{2} S^{2} \frac{\partial^{2} B}{\partial S^{2}}\right) d t
$$

where $\varphi_{b}$ is the recovery rate of the bond.

The PDE of the bond component is

$$
\frac{\partial B}{\partial t}+\frac{1}{2} \sigma^{2} S^{2} \frac{\partial^{2} B}{\partial S^{2}}+\left(r-q+h\left(1-\varphi_{s}\right)\right) S \frac{\partial B}{\partial S}-\left(r+h\left(1-\varphi_{b}\right)\right) B=0
$$

Equations (23) and (28) are coupled through appropriate final and boundary conditions reflecting the terms and conditions of each individual convertible and need to be solved simultaneously. Convertible bonds often incorporate various additional features, such as call and put provisions.

The final conditions at maturity $T$ can be generalized as 


$$
\begin{gathered}
G_{T}= \begin{cases}\eta S_{T}, & \text { if } \eta S_{T}>\min \left[P_{c}, \max \left(P_{p}, N+C\right)\right\rfloor \\
0, & \text { otherwise }\end{cases} \\
B_{T}=\left\{\begin{array}{cc}
\min \left[P_{c}, \max \left(P_{p}, N+C\right)\right\rfloor, & \text { if } \left.\eta S_{T} \leq \min \mid P_{c}, \max \left(P_{p}, N+C\right)\right\rfloor \\
0, & \text { otherwise }
\end{array}\right.
\end{gathered}
$$

where $N$ denotes the bond principal, $C$ denotes the coupon, $P_{c}$ denotes the call price, $P_{p}$ denotes the put price and $\eta$ denotes the conversion ratio. The final conditions tell us that the convertible bond at the maturity is either a debt or an equity.

The upside constraints at time $t \in[0, T]$ are

$$
\left\{\begin{array}{lr}
G_{t}=\eta S_{t}, B_{t}=0 & \text { if } \eta S_{t}>\min \left[P_{c}, \max \left(P_{p}, \tilde{L}_{t}\right)\right] \\
G_{t}=0, B_{t}=P_{p} & \text { else if } \tilde{L}_{t} \leq P_{p} \\
G_{t}=0, B_{t}=P_{c} & \text { else if } \tilde{L}_{t} \geq P_{c} \\
G_{t}=\tilde{G}_{t}, B_{t}=\tilde{B}_{t} & \text { else }
\end{array}\right.
$$

where $\widetilde{L}_{t}=\widetilde{B}_{t}+\widetilde{G}_{t}$ is the continuation value of the convertible bond, $\widetilde{B}_{t}$ is the continuation value of the bond component and $\widetilde{G}_{t}$ is the continuation value of the equity component. Equation (31) says that the convertible is either in the continuation region or one of the three constraints (called, put or converted). One can use finite difference methods to solve the PDEs (23) and (28) for the price of a convertible bond.

\section{Conclusion}

This paper aims to price hybrid financial instruments (e.g., convertible bonds) whose values may simultaneously depend on different assets subject to credit risk in a proper and consistent way. The motivation for our model is that if a company goes bankrupt, all the securities (including the equity) of the company default. The recovery is realized in accordance with the priority established by the Bankruptcy Code. In other words, different securities have the same probability of default, but different recovery rates.

We propose a hybrid framework to value risky equities and debts in a unified way. The model relies on the probability distribution of the default jump rather than the default jump itself. As such, the model can achieve a high order of accuracy with a relatively easy implementation. 


\section{Appendix}

In this section, we describe the numerical method used to solve discrete forms of (23) and (28). Let $x=\ln \left(\frac{S_{t}}{S_{0}}\right)$ and define backward time as $\delta=T-t$. The equations (23) and (28) can be rewritten as

$$
\begin{aligned}
& \frac{\partial B}{\partial \delta}-\frac{1}{2} \sigma^{2} \frac{\partial^{2} B}{\partial x^{2}}-\left(r-q+h\left(1-\varphi_{s}\right)-\frac{\sigma^{2}}{2}\right) \frac{\partial B}{\partial x}+\left(r+h\left(1-\varphi_{b}\right)\right) B=0 \\
& \frac{\partial G}{\partial \delta}-\frac{1}{2} \sigma^{2} \frac{\partial^{2} G}{\partial x^{2}}-\left(r-q+h\left(1-\varphi_{s}\right)-\frac{\sigma^{2}}{2}\right) \frac{\partial G}{\partial x}+\left(r+h\left(1-\varphi_{s}\right)\right) G=0
\end{aligned}
$$

The equations (A1) and (A2) can be approximated using Crank-Nicolson rule. We discretize the $x$ to be equally spaced as a grid of nodes $0 \sim M$. At the maturity, $G_{T}$ and $B_{T}$ are determined according to (29) and (30). At any time $i+1$, the boundary conditions are

$$
\begin{gathered}
\left\{\begin{array}{c}
B_{0}^{i+1}\left(1+0.5\left(r+h\left(1-\varphi_{b}\right) \Delta \tau\right)=B_{0}^{i}\left(1-0.5\left(r+h\left(1-\varphi_{b}\right)\right) \Delta \tau\right)\right. \\
G_{0}^{i+1}\left(1+0.5\left(r-q+h\left(1-\varphi_{s}\right) \Delta \tau\right)=G_{0}^{i}\left(1-0.5\left(r-q+h\left(1-\varphi_{s}\right)\right) \Delta \tau\right)\right.
\end{array}\right. \\
\left\{\begin{array}{c}
B_{M}^{i+1}=0 \\
G_{M}^{i+1}=\eta S_{M}
\end{array} \text { when } x \approx 0\right.
\end{gathered}
$$

\section{References}

Agarwal, V., Fung, W., Loon, Y. and Naik, N. (2007) Liquidity provision in the convertible bond market: analysis of convertible arbitrage hedge funds. CFR-working paper.

Ammann, M, Kind, A., and Wilde, C. (2008) Simulation-based pricing of convertible bonds. Journal of empirical finance, 15: 310-331.

Ayache, E., Forsyth, P. A., and Vetzal, K. R. (2003) The valuation of convertible bonds with credit risk. Journal of Derivatives 11: 9-30. 
Bloomberg (2009) OVCV model description. Quantitative research and development, Equities team.

Calamos, N. P. (2011) Convertible arbitrage: Insights and techniques for successful hedging. John Wiley \& Sons.

Carayannopoulos, P. and Kalimipalli, M. (2003) Convertible bond prices and inherent biases. Journal of Fixed Income 13: 64-73.

Davis, M. and Lischka, F. R. (1999) Convertible bonds with market risk and credit risk. Working paper, Tokyo-Mitsubishi International plc.

Duffie, D., and Singleton, K. J. (1999) Modeling term structure of defaultable bonds. Review of Financial Studies 12: 687-720.

FinPricing, 2013, Valuation service, https://finpricing.com/lib/EqRangeAccrual.html

Goldman Sachs (1994) Valuing convertible bonds as derivatives. Quantitative Strategies Research Notes, Goldman Sachs.

Jarrow, R. A. and Protter, P. (2004) Structural versus reduced form models: a new information based perspective. Journal of Investment Management 2: 34-43.

Merton, R. C. (1976) Option pricing when underlying stock returns are discontinuous. Journal of Financial Economy 3: 125-144. 
J.P. Morgan (2001) Par credit default swap spread approximation from default probabilities. Risk Publications.

Tsiveriotis, K. and Fernandes, C. (1998) Valuing convertible bonds with credit risk. Journal of Fixed Income 8: 95-102.

Zabolotnyuk, Y., Jones, R., and Veld, C., (2010), “An empirical comparison of convertible bond valuation models," Financial Management, 39, 2, 675-705. 